\title{
Variation of the Photoionization Cross-Section with the Position of a Hydrogenic Donor Impurity in a Gallium Arsenide Quantum Well Dot of Square Cross-Section
}

\author{
Winnie Otieno*, Hannington Oyoko \\ Department of Physics, Pwani University, Kilifi, Kenya \\ Email address: \\ winnieriana@gmail.com (W. Otieno),h.Oyoko@pu.ac.ke (H. Oyoko) \\ ${ }^{*}$ Corresponding author
}

To cite this article:

Winnie Otieno, Hannington Oyoko. Variation of the Photoionization Cross-Section with the Position of a Hydrogenic Donor Impurity in a Gallium Arsenide Quantum Well Dot of Square Cross-Section. American Journal of Optics and Photonics.

Vol. 6, No. 3, 2018, pp. 25-30. doi: 10.11648/j.ajop.20180603.11

Received: September 2, 2018; Accepted: October 6, 2018; Published: October 29, 2018

\begin{abstract}
In the present work, a theoretical study of the variation of the photoionization cross-section with the incident photon frequency and the axial position of a hydrogenic donor impurity in GaAs quantum well dot of square cross-section is carried out. In the calculation, a trial wave function in the effective mass approximation and a finite potential well is used. The wave function is constructed with an appropriate envelope wave function that satisfies the boundary conditions, i.e., the wave function vanishes at the boundary. A trial wave function is employed to calculate the total energy of the hydrogenic donor impurity in the ground state. The total energy is then minimized with respect to the variational parameter in the trial wave function to obtain the minimum energy. The minimized total energies are then used to determine the donor binding energies within the quantum dot. It is observed that for a quantum dot of constant cross-section, the binding energy increases with a decrease in dot length to a peak value; thereafter it decreases rapidly towards zero. The binding energies obtained are used to compute the photoionization cross-section of the hydrogenic donor impurity as a function of the incident photon frequency for different positions of the donor impurity. It is observed that the photoionization cross-sections rise steeply to their peaks from almost zero value then gradually decrease as the photon frequency increases until they become almost constant for very high photon frequencies. The photoionization cross-section peak is much higher for the hydrogenic donor impurity located closest to the centre of the quantum well dot than for donor impurity located farther away from the dot centre. This indicates that the photoionization cross-section is sensitive to the location of the donor impurity in the quantum dot and to the incident photon frequency.
\end{abstract}

Keywords: GaAsQuantum Dot, Hydrogenic Donor Impurity, Photoionization Cross-Section

\section{Introduction}

Semiconductor nano-structures can operate at their potential if they can be grown with high degree of purity and if any introduced impurities and defects are controlled. One of the ways of controlling the un-intentional impurities that are introduced to a semiconductor device is by photoionization. This makes photoionization cross-section an important optical property needed for characterization of a hydrogenic donor impurity in a semiconductor device. This is of great significance due to its numerous applications in laser design [1], astrophysics, radiation detection among others.
Photoionization cross-section of shallow donor impurities in nano-structures [2-5] have been studied theoretically by many researchers with the aim of improving opto-electronic device applications by reaching higher frequency devices, low power consumption devices and higher power generating devices. In particular, El-Said and Tomak [6, 7] have investigated the photon energy dependence of the photoionization cross-section of hydrogenic impurities in quantum wells [QW] using infinite barrier model. They found out that the photoionization cross-section depended on the polarization of the incident light relative to the direction of carrier confinement. Sali, et al [10] and Heon Ham, C. J. 
Lee and Harold N. Spector [17] using the infinite confining potential well model have investigated the photon energy dependence of the donor impurity along the growth axis $(\mathrm{z}-$ axis) of a hydrogenic donor in a GaAs quantum well wire [QWW] of cylindricalcross-section. They found that the cross-section rises steeply at the threshold frequency and then decreases with photon frequency. Variational procedure was used to study the influence of an axial magnetic field and hydrostatic pressure on the binding energy and the impurity related photoionization cross-section in quantum wire [24, 25]. The findings revealed that the photoionization crosssection strongly depends on the symmetry of the potential that confines the carriers. Later, Sahin et al [26] studied photoionization cross-section of an on-centrehydrogenic impurity in a multilayered spherical quantum dot. They used shooting method to determine electronic energy levels and their wave functions numerically. They observed that both the binding energy and the photoionization cross-section depended strongly on the layer thickness and photon energies. Based on the aforementioned observations that electronic and opto-electronic devices depend upon how electrons inside materials behave and how they are influenced by external perturbations which may be electrical [29-31], magneti c [32], electromagnetic, mechanical, hydrostatic pressure, this study has looked into the dynamic behavior of the photoionization cross-section. The variation of the photoionization cross-section with the incident photon frequency and with the position of the hydrogenic donor impurity in a square quantum well dot is determined using variational technique. The binding energy as a function of the dot length was determined. In addition the variation of the photoionization cross-section with axial position of hydrogenic donor impurity as a function of photon frequency has been investigated.

\section{Theoretical Framework}

In the calculation, the following Hamiltonian is used for a hydrogenic donor impurity placed along the axis of the GaAs quantum well.

$$
\hat{H}=-\frac{\hbar^{2}}{2 m^{*}}\left[\frac{\partial^{2}}{\partial x^{2}}+\frac{\partial^{2}}{\partial y^{2}}+\frac{\partial^{2}}{\partial z^{2}}\right]-\frac{e^{2}}{4 \pi \varepsilon_{0}} \frac{1}{\left[x^{2}+y^{2}+\left(z-z_{i}\right)^{2}\right]^{1 / 2}}+V_{B}(x, y, z)
$$

The quantity $\varepsilon 0$ is the dielectric constant of the system, $\mathrm{m} *$ is the effective mass, e is the elementary charge and $\mathrm{VB}$ confinement potential for the electron in the $\mathrm{x}, \mathrm{y}$ and $\mathrm{z}$ directions and is taken to be,

$$
V_{B}=\left\{\begin{array}{l}
0, \text { for }|x|,|y|,|z|<\frac{L_{x}}{2}, \frac{L_{y}}{2}, \frac{L_{z}}{2} \\
400 \mathrm{meV}, \text { elsewhewre }
\end{array}\right.
$$

The donor impurity is located at,

$$
\hat{r}=\left[x^{2}+y^{2}+\left(z-z_{i}\right)^{2}\right]^{1 / 2}
$$

The method of solution of Schrodinger equation is based on the variational principle within the effective mass approximation and the following trial wave function is used

$$
\psi_{1 s}(x, y, z)=N_{1 s} \cos (\alpha x) \cos (\beta y) \cos (\lambda z) \exp \left\{-\delta\left[x^{2}+y^{2}+\left(z-z_{i}\right)^{2}\right]^{1 / 2}\right\}
$$

In the above equation, $\delta$ is a variational parameter, $\alpha, \beta$ and $\lambda$ are constants while $N_{1 s}$ the normalization constant. The total energy $\langle\hat{H}\rangle$ has been calculated using the Equations (1) and (4),

$$
\langle\hat{H}\rangle=\frac{\left\langle\psi_{1 s}|\hat{H}| \psi_{1 s}\right\rangle}{\left\langle\psi_{1 s} \mid \psi_{1 s}\right\rangle}
$$

The total energy was then minimized to obtain the minimum energy Eminsubject to the condition,

$$
\begin{aligned}
& \frac{\partial\langle\hat{H}\rangle}{\partial \delta}=0 \\
& \sigma(\hbar \omega)=\left[\left(\frac{\xi_{e f f}}{\xi_{0}}\right)^{2} \frac{n_{r}}{\varepsilon}\right] \frac{4 \pi^{2}}{3} \alpha_{F s} \hbar \omega \times \sum_{f}\left|\left\langle\psi_{i}|\vec{r}| \psi_{f}\right\rangle\right|^{2} \delta^{\prime}\left(E_{f}-E_{i}-\hbar \omega\right)
\end{aligned}
$$

The minimum energy is then subtracted from the free energy, Eo, to obtain the binding energyEb [18-19].

$$
E_{\text {binding }}=E_{0}-E_{\min }
$$

Where Eo is the free energy, i.e., the energy in the absence of the hydrogenic donor impurity. It is given by,

$$
E_{0}=\frac{\hbar^{2}}{2 m^{*}}\left(\alpha^{2}+\beta^{2}+\lambda^{2}\right)
$$

The binding energy obtained from the above is then employed to calculate the photoionization cross-section [20, 
This expression describes photoionization cross-section for the transition of the donor impurity from ground state to a final state. The lifetime of the donor impurity in its initial state is given by summing up all possible final states,

$$
\frac{1}{\eta}=\frac{2 \pi}{\hbar} \sum_{f}\left|\left\langle\psi_{f}|\hat{r}| \psi_{i}\right\rangle\right|^{2} \delta\left(E_{f}-E_{i}\right)
$$

where $\mathrm{nr}$ is the refractive index of the semiconductor, $\varepsilon$ the dielectric constant of the medium[18].

$\alpha_{F s}=\frac{e^{2}}{\hbar c}$, is the fine structure constant and $\hbar \omega$ is the

$$
\delta^{\prime}\left(E_{f}-E_{i}-\hbar \omega\right)=\frac{\hbar \Gamma}{\pi\left[\left(\hbar \omega-\left(E_{f}-E_{i}\right)\right)^{2}+(\hbar \Gamma)^{2}\right]}
$$

Here, $\Gamma$ is the hydrogenic impurity line width and taken as $0.1 R_{y}^{*} . R_{y}^{*}$ is the effective Rydberg energy which is approximately $5.25 \mathrm{meV}$. The final state wave function is given by,

$$
\psi_{f}(x, y \cdot z)=N_{f} \cos (\alpha x) \cos (\beta y) \cos (\lambda z) \exp (-i k z)
$$

And its normalization constant given as,

$$
N_{f}^{2}=\left[\int_{0}^{L_{x}} \cos ^{2}(\alpha x) d x \int_{0}^{L_{y}} \cos ^{2}(\beta y) d y \int_{0}^{L_{z}} \cos ^{2}(\lambda z) \exp (-2 i k z) d z\right]^{-1}
$$

where $L_{z}$ is the length of the quantum well dot and $\mathrm{kz}$ is the one dimensional wave vector of the electron along the axis of the well. The final state energy into which the hydrogenic donor impurity scatters is given by,

$$
E_{f}=\frac{\hbar^{2} N_{f}^{2}}{2 m^{*}}\left(\alpha^{2}+\beta^{2}+\lambda^{2}-k^{2}\right) \int_{0}^{l_{x}} \cos ^{2}(\alpha x) d x \int_{0}^{l y} \cos ^{2}(\beta y) d y \int_{0}^{l_{z}} \cos ^{2}(\lambda z) d z-\frac{e^{2} N_{f}^{2}}{4 \pi \varepsilon_{0}} \int_{0}^{l_{x}} \cos ^{2}(\alpha x) d x \int_{0}^{l_{y}} \cos ^{2}(\beta y) d y \int_{0}^{\frac{l_{z}}{2}} \frac{\cos ^{2}(\lambda z) d z}{\left[x^{2}+y^{2}+z^{2}\right]^{\frac{1}{2}}}(14)
$$

where $\mathrm{k}$ depends on the final state into which the donor impurity scatters [22]. The matrix element $\sum_{f}\left|\left\langle\psi_{i}|\vec{r}| \psi_{f}\right\rangle\right|^{2}$ in the photoionization cross-section equation 9 is given by;

$$
\left[N_{i} N_{f} \int_{0}^{L_{x}} \cos ^{2}(\alpha x) d x \int_{0}^{L_{y}} \cos ^{2}(\beta y) d y \int_{0}^{\frac{l_{z}}{2}}|\vec{z}| \cos ^{3}(\lambda z) \exp \left(-\delta\left\{x^{2}+y^{2}+\left(z-z_{i}\right)^{2}\right\}^{\frac{1}{2}}+i k z\right) d z\right]^{2}
$$

Where $\vec{r}=\hat{z} \cos (\lambda z)$

\section{Results and Discussion}

Binding energy

In figure 1, binding energy peaks at $57.0984 \mathrm{meV}$ at relatively small dot length value of about $18.7326 \mathrm{~nm}$ and then decreases with further increase in dot length. It is also noted that as the well length increases, the binding energy of the hydrogenic donor impurity tends towards a constant value of about $44.0 \mathrm{meV}$. This compares favorably with that found by Correa, et al [27]. That is, the binding energy is a nonmonotonic function of the well width and peaks at a relatively small width value. This is because where the electron distribution is more localized, the probability of finding the electrons around the impurity is increased hence the energy levels are pulled down because of the attractive coulomb potential of the hydrogenic donor impurity while at larger dot length where the electrons feels less attractive effect of the hydrogenic donor impurity, the binding energy becomes small. 


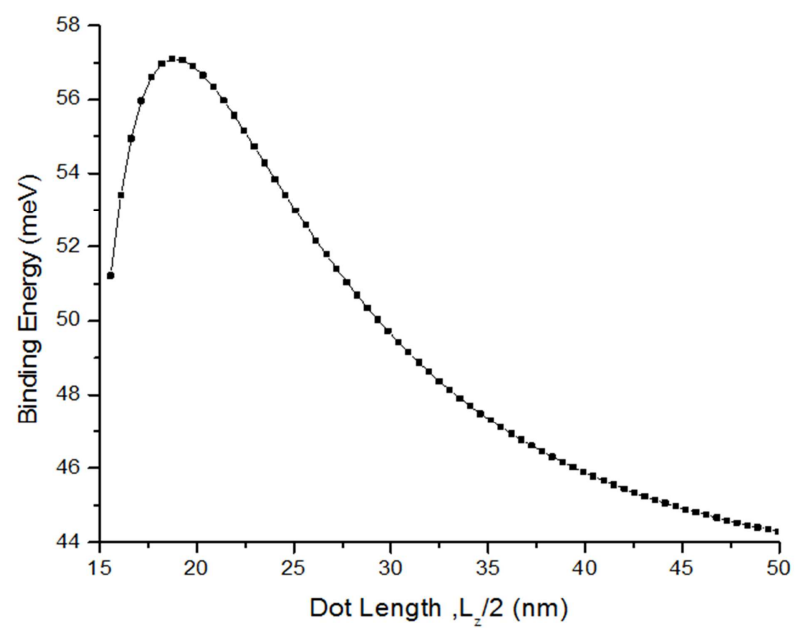

Figure 1. The donor impurity binding energy is shown as a function of the dot length in the 100nm GaAs quantum well dot embedded in a matrix of $G a_{1-x} A l_{x} A s$.

Variation of photoionization cross-section with axial position of hydrogenic donor impurity

Figure 2 shows a graph of photoionization cross-section as a function of photon frequency for a donor impurity located at $z_{i}=10 \mathrm{~nm}$ from the dot centre. It is observed that the photoionization cross-section increases rapidly with increasing incident photon frequency up to a peak of $\sigma=3.5 \times 10^{-14} \mathrm{~cm}^{2}$ at about $\omega=1.75 \mathrm{THz}$, then decreases almost exponentially to zero at about $\omega=17 \mathrm{THz}$ for a quantum well dot of length $L_{z}=100.0 \mathrm{~nm}$.

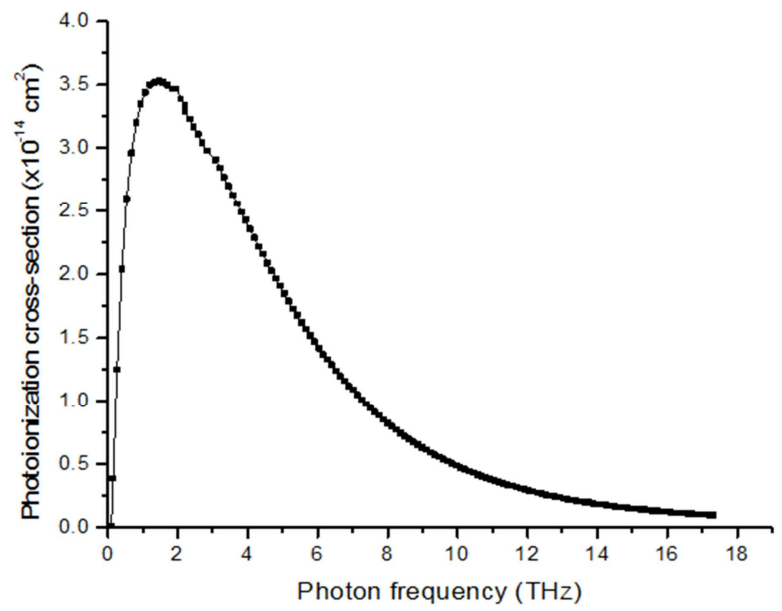

Figure 2. Photoionization cross-section is shown as a function of incident photon frequency for the donor impurity located at $z_{i}=10.0 \mathrm{~nm}$ from the centre of the GaAs quantum well dot with finite confining potential.

Where as the overlap of two distinct initial and final state wave functions in the computation of matrix element is often only partial; in this case the overlap of wave functions for the inter-sublevels is higher than the usual situation hence the electron tunnels easily resulting to a large photoionization cross-section.

In Figure 3, the donor impurity is located at $z_{i}=25.0 \mathrm{~nm}$.

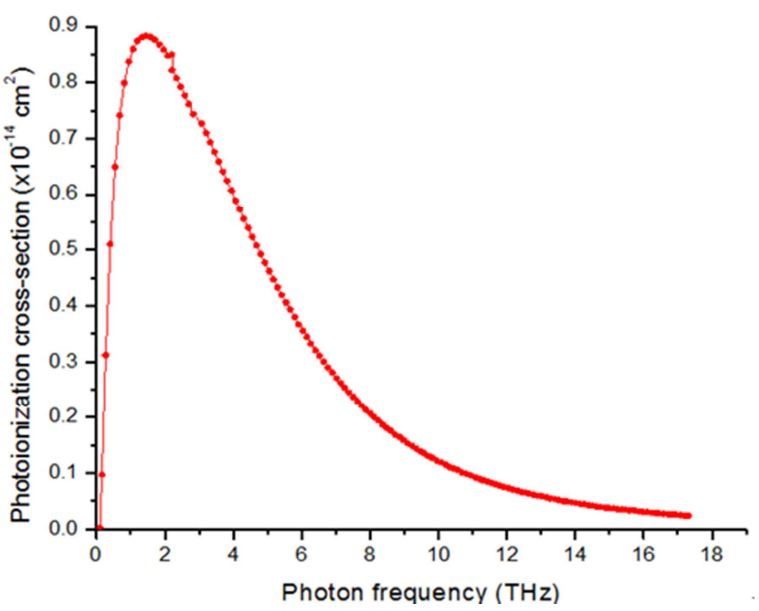

Figure 3. Photoionization cross-section is shown as a function of incident photon frequency for the donor impurity located at $z_{i}=25.0 \mathrm{~nm}$ from the centre of the GaAs quantum dot with finite confining potential.

Here the photoionization cross-section rises rapidly up to a peak of only $\sigma=0.9 \times 10^{-14} \mathrm{~cm}^{2}$ at a frequency of about $\omega=1.25 \mathrm{THz}$. It then decreases much more gradually than the first curve to a flat value of nearly zero from about $\omega=15 \mathrm{THz}$. In this case, the barrier thickness is increased so that the overlapping of the wave functions between the initial and final state wave functions decreases because of the localization of the electrons hence the photoionization crosssection decreases.

In Figure 4, the donor impurity is located at $z_{i}=45.0 \mathrm{~nm}$ and we observed an even smaller peak of about $\sigma=0.5 \times 10^{-14} \mathrm{~cm}^{2}$ at about $\omega=1.5 \mathrm{THz}$. This then gradually decreased to zero at about $\omega=15 \mathrm{THz}$. In this case, the dot region is big, making the tunneling not easy and the electron is confined almost completely in the core region hence a smaller photoionization cross-section.

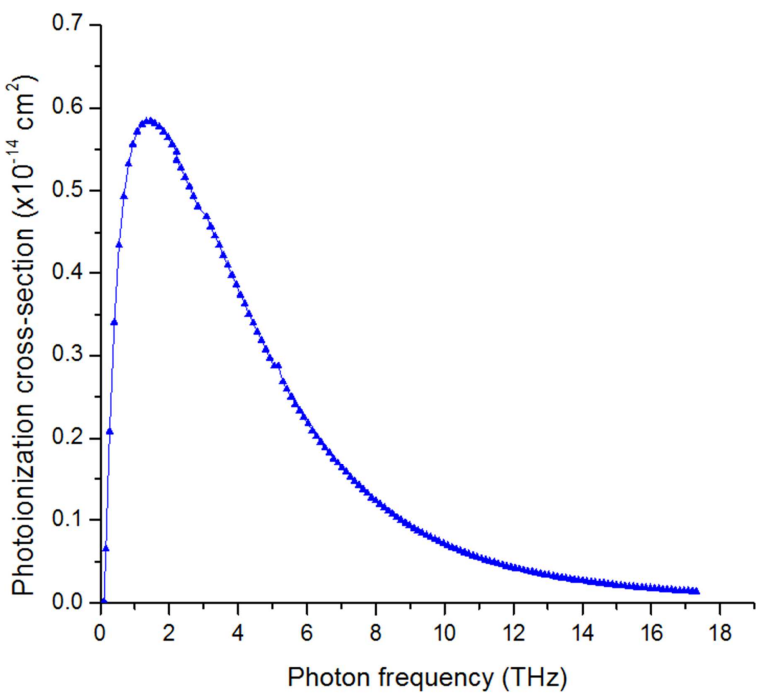

Figure 4. Photoionization cross-section is shown as a function of incident photon frequency for the donor impurity located at $z_{i}=45.0 \mathrm{~nm}$ from the centre of the GaAs quantum dot with finite confining potential.

In Figure 5, the photoionization cross-sections are shown 
as functions of the photon frequencies for the three different impurity positions $z_{i}=10 \mathrm{~nm}, 25 \mathrm{~nm}$ and $45 \mathrm{~nm}$ in a GaAs quantum well dot using finite dot model for on-axis impurities. It is observed that from the three positions of the donor impurities, the variation of the photoionization crosssection with photon frequency is very similar.

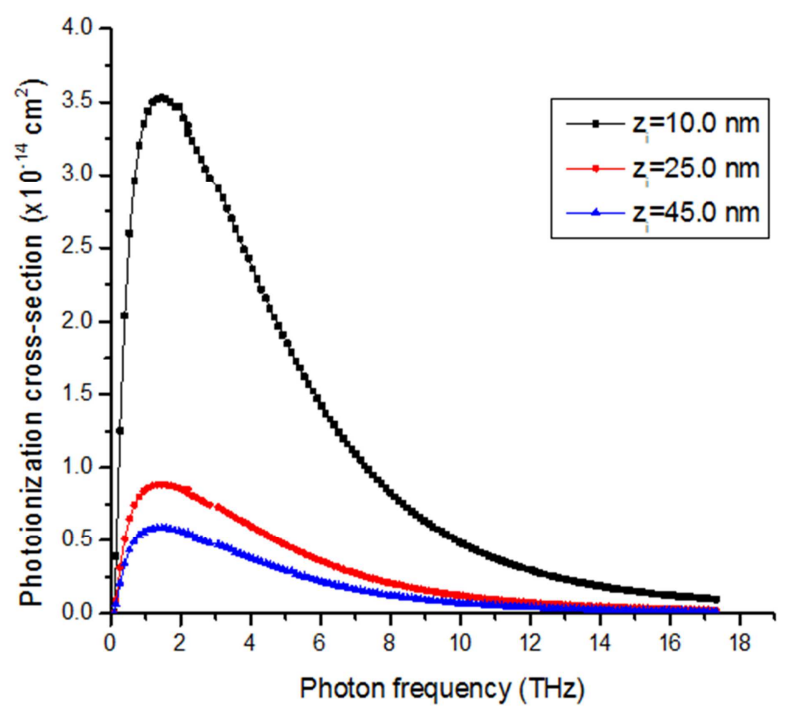

Figure 5. Photoionization cross-section is shown as a function of incident photon frequency for different positions of the donor impurity along the growth axis (z) within the GaAs quantum dot with finite confining potential.

It is also noted that at low photon frequencies, the photoionization cross-section falls rapidly to zero. This is indicative of the sublevels separation, $E_{f}-E_{i}$, being less than the photon energy $\hbar \omega$. It also indicates that the photoionization cross-section is insensitive to frequencies below a certain threshold. This occurs as the electrons in upper sublevels do not have sufficient energy to emit a photon and hence are unable to be photoionized. Photoionization only occurs for resonance condition, i.e., when the sublevel separation is equal to photon energy and the dipole matrix element $\sum_{f}\left|\left\langle\psi_{f}|\vec{r}| \psi_{i}\right\rangle\right|^{2}$ now plays an effective role on the photoionization cross-section. In the three cases, the peak value of the cross-section occurs at lower photon frequencies since at lower photon frequencies, the final state for photoionization is the free electron state associated with the first sublevel in the dot, $\hbar \omega=E_{\text {binding }}$ [23]. Thus the farther the donor impurity is located from the centre of the quantum dot the smaller is the photoionization cross-section. The results are in agreement with the reported theoretical estimates by Sali, et al [10]. We also noted that decreasing the size of the integration step length $\delta l$ (which will increase the accuracy of any numerical integrals) increases the subsequent peak values of the photoionization cross-section by a factor of around 10. The step through $\delta l=\lambda / 10$ is considered as a good compromise for all subsequent calculations. The spread of the photoionization cross-section was attributed to the free motion of the donor impurity along the z-direction and the fact that the binding energy of hydrogenic donor impurity increases with decrease in dot-length in the finite dot model. This indicates that the photoionization cross section is sensitive to the location of the donor impurity in the quantum dot.

\section{Conclusion}

The variation of photoionization cross-section with the position of the donor impurity in a quantum well dot is calculated. The results show that photoionization crosssection is much larger for donor impurities located close to the centre of the quantum well dot than for those farther away from the quantum well dot centre. Furthermore, it is found that the photoionization cross-section rises steeply at lower photon frequency and then gradually decreases to almost constant values for higher photon frequencies. Since both the sublevel energies and the donor impurity binding energy increase with decreasing dot length, the frequency at which the photoionization cross-section peaks increases with decreasing dot length and by changing the donor impurity position, a large spread in the photoionization cross-section is obtained. It is further shown that the photoionization crosssection can be controlled by adjusting the position of the hydrogenic donor impurity in a quantum dot of square crosssection and that the sensitivity of an opto-electronic device can also be tuned with the variation of photoionization crosssection with the position of the hydrogenic donor impurity. The results show that the distance of the hydrogenic donor impurity from the dot centre has an almost is inverse proportion relation to the photoionization cross-section which is important since some opto-electronic devices are sensitive to large photoionization cross-section while some are sensitive to small photoionization cross-section. Hence, in designing opto-electronic devices, one needs to consider the location of any hydrogenic donor impurities that are embedded within a quantum well dot. The results are in agreement with the recently reported theoretical estimates. It is hoped that this present work will provide a stimulus to other researchers in the field to fill the gaps that may become apparent later.

\section{Acknowledgements}

The authors are grateful to Dr. Fredrick Mutunga of Pwani University for numerical assistance and to Dr. George Manyali of Masinde Muliro University for his valuable comments and suggestions.

\section{References}

[1] Radu, A., Kirakosyan, A. A., Laroze, D., \& Barseghyan, M. G., Semiconductor Science and. Technology, 30 (4), 045006, 2015.

[2] Porras-Montenegro N \& Perez-Merchancano S T, Phys Rev B, 46, 9780, 1992. 
Winnie Otieno and Hannington Oyoko: Variation of the Photoionization Cross-Section with the Position of a Hydrogenic Donor Impurity in a Gallium Arsenide Quantum Well Dot of Square Cross-Section

[3] Porras-Montenegro N \& Perez-Merchancano S T \& Latge'A, J Appl Phys. 74, 7624, 1993.

[4] Brown J W \& Spector H N, J ApplPhys, 59, 1179, 1986.

[5] Takikawa M, Kelting K, Barunthaler G, et al, J. Appl. Phys, 65, 3937, 1989.

[6] El-Said M \&Tomak M, J Phys Chem Solids, 52, 603, 1991.

[7] El Said M \&Tomak M, Solid State Commun, 82, 721, 1993.

[8] Ilaiwi K F \& El-said M, Phys Stat Sol B, 187, 93, 1995.

[9] Jayam G Sr\&Navaneethakrishnan K, Solid State Commun, $122,433,2002$.

[10] Sali A, Fliyou M, Roubi L \&Loumrhari H, J PhysCondens Matter, 11, 2427, 1999.

[11] Sali A, Fliyou M, Satori H \&Loumrhari H, Phys Status Solidi B, 211, 661, 1999.

[12] Sali A, Fliyou M \& Loumrhari H, Physica B, 233, 196, 1997.

[13] Sali A, Satori H, Fliyou M \&Loumrhari, Phys Status Solidi B, 200, 145, 1997.

[14] Sali A, Fliyou M, \& Loumrhari H, Phys Status Solidi B, 232, 209, 2002.

[15] Sali A, Fliyou M, Satori H \&Loumrhari H, J Chem Solids, 64, $31,2003$.

[16] Heon H \& Lee J L, J Korean PhysSoc, 42, S289, 2003.

[17] J Lee and H N Spector, J VacSciTechnol B, 16, 2, 1984.

[18] Heon H, Lee C J \& Spector H N, J ApplPhys, 196, 2004.
[19] Oyoko H \&Csavinzsky P, Phys Rev B, 43, 9263, 1991.

[20] Lax M, In the Proceedings of the 1954 Atlantic City Conference on Photocunductivity, Wiley, New York, 111, 1956.

[21] Arfken G, Mathematical Methods for Physicists, 3rd Edition, Academic Press, NewYork, 1985.

[22] Bastard G, Phys Rev B, 24, 4714, 1981.

[23] Heon H, \& Spector, H. N., J Appl Phys, 97, 3900-3905, 2003.

[24] Barseghyan, M. G., Kirakosyan, A. A, \& Duque, C. A. Physics Satus Solidi B, 246, 626-629, 2009.

[25] Correa, J. D., Cepeda-giraldo, O., Porras-Montenegro, N., \& Duque, C. A. Physics Satus. Solidi B, 241, 3311-3317, 2004.

[26] Sahin, M., Tek, F., \& Erdine, A. Journal of Applied Physics, 111, (2012)1-17.

[27] Correa, J. D., Porras-Montenegro, N. \& Duque, C. A., Brazilian Journal of Physics 2A, 36, 387-390, 2006.

[28] Ghosh, A. P., Mandal, A., Sarkar, S., \&Ghosh, M., Optics Communications, 367, 325-334, 2016.

[29] Tshipa M. \& Masale M., intechopen, 75736, 2018.

[30] Tshipa M, Condensed Matter Physics, 20 (2), 23703, 2017.

[31] Wang, G., Duan, X., \& Chen, W, journal of nanomaterials, 8(7), 240563, 2013.

[32] Yun, K., Sheng, W., \& Xianli, L., journal of semiconductors, 36(3), 32003, 2015. 